\title{
Evaluation of Reliability and Availability Characteristics of 2-out of -3 Standby System under a Perfect Repair Condition
}

\author{
Ibrahim Yusuf*, Nafiu Hussaini \\ Department of Mathematical Sciences, Faculty of Science, Bayero University, Kano
}

\begin{abstract}
Many authors have studied the effectiveness of a redundant system under two or three types of failure under the assumption that such failures are repairable. Little attention is paid on whether such repair action can restore the system operating condition to as good as new (perfect repair) and the effect of such perfect repair on the system performance. In this study, various measures of system effectiveness such as mean time to system failu re (MTSF), steady state availability, busy period and profit function of a 2-out-of-3 repairable system with perfect repair are analy zed using Kolmogorov's forward equation method. Some particular cases have been discussed graphically. The results have indicated that perfect repair action plays a vital role on system performance. Simulations results show that perfect repair is important particularly in increasing mean time to system failure, availability and system performance as a whole.
\end{abstract}

Keywords Mean Time To System Failure (MTSF), Availability, Profit, Redundancy, Perfect Repair, Kolmogorov's Equation Method, 2-Out-Of-3 Standby System

\section{Introduction}

Redundancy is a technique used to improve system reliability and availability. Reliability is vital for proper utilization and maintenance of any system. It involves technique for increasing system effectiveness through reducing failure frequency and maintenance costmin imizati on. One of the forms of redundancy is the k-out-of-n system which finds wide application in industrial system. Redundancy, repair action (perfect), and preventive maintenance are some of the ways by which the reliability of a system can be improved. K-out-of-n systems are often encountered industrial applications. Electronics industry, telecommunication network systems, power generator and transmission systems are the common examples ofk-out-ofn systems. In general, there are three types in standby, i.e. cold, hot and warm standby. A lot of papers dealing with the reliability and availability of k-out-of-n systems (standby systems) have been published.[3] discussed cost analysis of k-out-of-n: G system with active standby redundancy components and repair facility,[2] studied the cost analysis of a two unit cold standby redundant system with two types of unit failure involving common cause failure,[1] deals with

* Corresponding author:

ibrahimyusif@yahoo.com (Ibrahim Yusuf)

Published online at http://journal.sapub.org/ajms

Copyright (C) 2012 Scientific \& Academic Publishing. All Rights Reserved cost analysis of a two unit cold standby redundant system with two types of failure and preventive maintenance,[4] studied profit analysis of a two unit cold standby systemwith preventive maintenance,[6] deals with stochastic analysis of a redundant system involving common human failure,[5] deals with the evaluation of reliability and availability characteristics of different system.[7] studied cost analys is of series systems with cold standby components and a repairable service station, Bhatti et al[8], considered two identical unit cold standby systems with single repairman. Kumar et al[9] analy zed cost benefit analysis of a two unit system in which units work in parallel and become degraded after repair, Gupta el al[10] deals with stochastic analysis of a two non identical unit standby model. The system consist priority unit (p) and ordinary unit (o). A single repair facility appears and disappears from the system randomly with constant rates, Malik et al[11] analyzed two reliability models for a system of non identical units original and duplicate using regenerative point technique., Mahmoud and Moshrefa[12] deal with the study of the stochastic analysis of a two unit cold standby system considering hardware failure, human error failure and preventive maintenance, Yusuf and Bala[13], studied stochastic two models of two unit parallel system. In model I, the system can be normal, deterioration (slow, mild or fast deterioration), failure whereas in model II, the system can either be in normal of failure modes. Using linear first order linear differential equations, various measures of system effectiveness such as mean time to system failure (MTSF) and availability are 
obtained to see the effect of deterioration on such measures, Kumar and Kadyan[14] deal with profit analysis of two unit non identical system with degradation and rep lacement while Sureria et al[15] studied cost benefit analysis of a computer system with priority to software replacement over hardware repair.

\subsection{Objectives}

Many authors studied redundant system exposes to different failure types. However, such systems are subject to different types of repairs like as bad as old, imperfect repair, worse repair, worst repair meaning that in course of time their condition falls from higher to lower, and subsequently fail. However some repairs (perfect) can restore the system operating condition to as good as new The question raise here is whether such perfect repairs can affect some measures of system effectiveness like mean time to system failure (MTSF) and system availability. The purpose of the present paper is to discuss reliability and availability evaluation of 2-out-of-3 system under perfect repair condition. The system is failed if two of its units failed. The system is analyzed using Kolmogorov's equations method. Graphs are plotted to highlight important results.

\section{Notations}

$\beta_{i}$ Constant type $i$ failure rate $\alpha_{i}$ Constant type $i$ repair rate

$F_{R i}$ Failed unit under type $i$ repair $F_{W i}$ Failed unit waiting for type $i$ repair $i=1,2,3$

$\mathrm{S}$ Unit in Standby O Unit in operation

\section{Assumptions}

1. The system consist of 3 components/units

2. Initially two units are in operable condition of full capacity

3. The system is failed when the number of working component goes down below 2

4. Failure and repair time follow exponential distribution

5. Repair is as good as new(Perfect repair).

\section{Method}

\section{State of the System}

Fig. 1 Schematic diagram of the system

$$
\begin{array}{ll}
S_{0}\left(O_{1}, O_{2}, O_{S}\right), & S_{1}\left(F_{R 1}, O_{2}, O_{3}\right), S_{2}\left(O_{1}, F_{R 2}, O_{3}\right), \\
S_{3}\left(F_{R 1}, O_{2}, F_{W 3}\right), & S_{4}\left(O_{1}, O_{2}, F_{R 3}\right), S_{5}\left(F_{R 1}, F_{W 2}, O_{3}\right), \\
S_{6}\left(O_{1}, F_{R 2}, F_{W 3}\right) &
\end{array}
$$

\subsection{Mean Time to System Failure Analysis}

From Fig. 1 above, define $P_{i}(t)$ to be the probability that the system at time $t,(t \geq 0)$ is in state $S_{i}$. Let $P(t)$ be the probability row vector at time $t$, the initial condition for this paper are

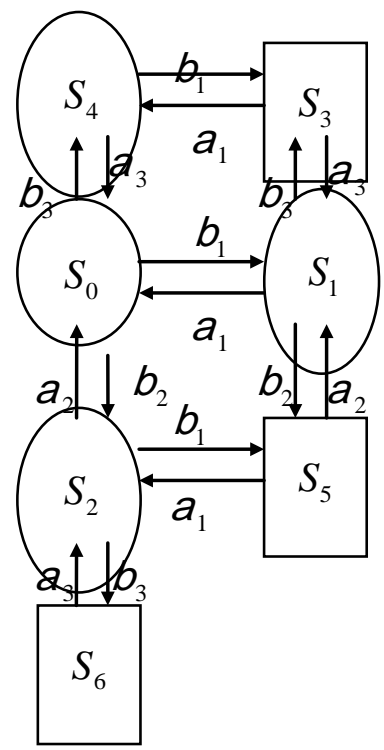

Figure 1. Schematic diagram of the system

$$
\begin{aligned}
P(0) & =\left[P_{0}(0), P_{1}(0), P_{2}(0), P_{3}(0), P_{4}(0), P_{5}(0), P_{6}(0)\right] \\
& =[1,0,0,0,0,0,0]
\end{aligned}
$$

we obtain the following differential equations:

$$
\begin{aligned}
& \frac{d P_{0}(t)}{d t}=-\left(\beta_{1}+\beta_{2}+\beta_{3}\right) P_{0}(t)+\alpha_{1} P_{1}(t)+\alpha_{2} P_{2}(t)+\alpha_{3} P_{4}(t) \\
& \frac{d P_{1}(t)}{d t}=-\left(\alpha_{1}+\beta_{2}+\beta_{3}\right) P_{1}(t)+\beta_{1} P_{0}(t)+\alpha_{3} P_{3}(t)+\alpha_{2} P_{5}(t) \\
& \frac{d P_{2}(t)}{d t}=-\left(\alpha_{2}+\beta_{1}+\beta_{3}\right) P_{2}(t)+\beta_{2} P_{0}(t)+\alpha_{1} P_{5}(t)+\alpha_{3} P_{6}(t) \\
& \frac{d P_{3}(t)}{d t}=-\left(\alpha_{1}+\alpha_{3}\right) P_{3}(t)+\beta_{3} P_{1}(t)+\beta_{1} P_{4}(t) \\
& \frac{d P_{4}(t)}{d t}=-\left(\alpha_{3}+\beta_{1}\right) P_{4}(t)+\beta_{3} P_{0}(t)+\alpha_{1} P_{3}(t) \\
& \frac{d P_{5}(t)}{d t}=-\left(\alpha_{1}+\alpha_{2}\right) P_{5}(t)+\beta_{2} P_{1}(t)+\beta_{1} P_{2}(t) \\
& \frac{d P_{5}(t)}{d t}=-\left(\alpha_{1}+\alpha_{2}\right) P_{5}(t)+\beta_{2} P_{1}(t)+\beta_{1} P_{2}(t) \\
& \frac{d P_{6}(t)}{d t}=-\alpha_{3} P_{6}(t)+\beta_{3} P_{2}(t)
\end{aligned}
$$

the system of differential equations can be written in matrix as form as $\dot{P}=A P$

Where 


$$
A=\left[\begin{array}{ccccccc}
-\left(\beta_{1}+\beta_{2}+\beta_{3}\right) & \alpha_{1} & \alpha_{2} & 0 & \alpha_{3} & 0 & 0 \\
\beta_{1} & -\left(\alpha_{1}+\beta_{2}+\beta_{3}\right) & 0 & \alpha_{3} & 0 & \alpha_{2} & 0 \\
\beta_{2} & 0 & -\left(\alpha_{2}+\beta_{2}+\beta_{3}\right) & 0 & 0 & \alpha_{1} & \alpha_{3} \\
0 & \beta_{3} & 0 & -\left(\alpha_{1}+\alpha_{3}\right) & \beta_{1} & 0 & 0 \\
\beta_{3} & 0 & 0 & \alpha_{1} & -\left(\alpha_{3}+\beta_{1}\right) & 0 & 0 \\
0 & \beta_{2} & \beta_{1} & 0 & 0 & -\left(\alpha_{1}+\alpha_{2}\right) & 0 \\
0 & 0 & \beta_{3} & 0 & 0 & 0 & -\alpha_{3}
\end{array}\right]
$$

It is difficult to evaluate the transient solutions hence following $[3,4,7]$ we delete the rows and colu mns of absorbing state of matrix $A$ and take the transpose to produce a new matrix, say $Q$.

The expected time to reach an absorbing state is obtained from

$$
M T S F=P(0)\left(-Q^{-1}\right)\left[\begin{array}{l}
1 \\
1 \\
1 \\
1
\end{array}\right]
$$

Where

$$
Q=\left[\begin{array}{cccc}
-\left(\beta_{1}+\beta_{2}+\beta_{3}\right) & \beta_{1} & \beta_{2} & \beta_{3} \\
\alpha_{1} & -\left(\alpha_{1}+\beta_{2}+\beta_{3}\right) & 0 & 0 \\
\alpha_{2} & 0 & -\left(\alpha_{2}+\beta_{1}+\beta_{3}\right) & 0 \\
\alpha_{3} & 0 & 0 & -\left(\alpha_{3}+\beta_{1}\right)
\end{array}\right]
$$

The steady state mean time to system failure is:

$$
\begin{gathered}
M T S F=\frac{N_{1}}{D_{1}} \\
N_{1}=\left(\alpha_{1}+\beta_{2}+\beta_{3}\right)\left(\alpha_{2}+\beta_{1}+\beta_{3}\right)\left(\alpha_{3}+\beta_{1}\right)+\beta_{1}\left(\alpha_{2}+\beta_{1}+\beta_{3}\right)\left(\alpha_{3}+\beta_{1}\right)+\beta_{2}\left(\alpha_{1}+\beta_{2}+\beta_{3}\right)\left(\alpha_{3}+\beta_{1}\right)^{+} \\
\beta_{3}\left(\alpha_{1}+\beta_{2}+\beta_{3}\right)\left(\alpha_{2}+\beta_{1}+\beta_{3}\right) \\
D_{1}=\alpha_{3} \beta_{1} \beta_{2}^{2}+3 \beta_{1}^{2} \beta_{2} \beta_{3}+2 \beta_{1} \beta_{2} \beta_{3}^{2}+\alpha_{3} \beta_{2}^{2} \beta_{3}+\beta_{1} \beta_{2}^{2} \beta_{3}+\alpha_{2} \beta_{1} \beta_{3}^{2}+\alpha_{3} \beta_{1}^{2} \beta_{3}+\beta_{1}^{3} \beta_{3}+\alpha_{1} \beta_{1}^{2} \beta_{2}+2 \alpha_{3} \beta_{1} \beta_{2} \beta_{3}+ \\
\alpha_{2} \alpha_{3} \beta_{1} \beta_{2}+\alpha_{3} \beta_{2} \beta_{3}^{2}+\beta_{1} \beta_{3}^{3}+\alpha_{3} \beta_{1} \beta_{3}^{2}+2 \beta_{1}^{2} \beta_{3}^{2}+\alpha_{2} \beta_{1}^{2} \beta_{3}+\alpha_{1} \alpha_{3} \beta_{2} \beta_{3}+\alpha_{2} \beta_{1} \beta_{2} \beta_{3}+\alpha_{2} \alpha_{3} \beta_{1} \beta_{3}+ \\
\alpha_{1} \beta_{1} \beta_{2} \beta_{3}+\alpha_{1} \alpha_{2} \beta_{1} \beta_{3}+\alpha_{1} \alpha_{3} \beta_{1} \beta_{2}+\alpha_{3} \beta_{1}^{2} \beta_{2}+\beta_{1}^{2} \beta_{2}^{2}+\beta_{1}^{3} \beta_{2}+\alpha_{2} \beta_{1}^{2} \beta_{2}+\alpha_{1} \beta_{1}^{2} \beta_{3}+\alpha_{1} \beta_{1} \beta_{3}^{2}
\end{gathered}
$$

\subsection{Availability Analysis}

For the analysis of availability case of Fig. 1 using the same in itial conditions for this problem as

$$
P(0)=\left[P_{0}(0), P_{1}(0), P_{2}(0), P_{3}(0), P_{4}(0), P_{5}(0), P_{6}(0)\right]=[1,0,0,0,0,0,0]
$$

The differential equations can be expressed as

$$
\left[\begin{array}{c}
\dot{P}_{0} \\
\dot{P_{1}} \\
\dot{ \pm} \\
P_{2} \\
\dot{P_{3}} \\
\dot{P_{4}} \\
\dot{P_{5}} \\
\dot{P_{6}}
\end{array}\right]=\left[\begin{array}{ccccccc}
-\left(\beta_{1}+\beta_{2}+\beta_{3}\right) & \alpha_{1} & \alpha_{2} & 0 & \alpha_{3} & 0 & 0 \\
\beta_{1} & -\left(\alpha_{1}+\beta_{2}+\beta_{3}\right) & 0 & \alpha_{3} & 0 & \alpha_{2} & 0 \\
\beta_{2} & 0 & -\left(\alpha_{2}+\beta_{2}+\beta_{3}\right) & 0 & 0 & \alpha_{1} & \alpha_{3} \\
0 & \beta_{3} & 0 & -\left(\alpha_{1}+\alpha_{3}\right) & \beta_{1} & 0 & 0 \\
\beta_{3} & 0 & 0 & \alpha_{1} & -\left(\alpha_{3}+\beta_{1}\right) & 0 & 0 \\
0 & \beta_{2} & \beta_{1} & 0 & 0 & -\left(\alpha_{1}+\alpha_{2}\right) & 0 \\
0 & 0 & \beta_{3} & 0 & 0 & 0 & -\alpha_{3}
\end{array}\right]\left[\begin{array}{l}
P_{0} \\
P_{1} \\
P_{2} \\
P_{3} \\
P_{4} \\
P_{5} \\
P_{6}
\end{array}\right]
$$

The steady-state availability is given by 


$$
A(\infty)=P_{0}(\infty)+P_{1}(\infty)+P_{2}(\infty)+P_{4}(\infty)
$$

In the steady state, the derivatives of the state probabilities become zero so that

$$
A P=0
$$

which in matrix form

$$
\left[\begin{array}{ccccccc}
-\left(\beta_{1}+\beta_{2}+\beta_{3}\right) & \alpha_{1} & \alpha_{2} & 0 & \alpha_{3} & 0 & 0 \\
\beta_{1} & -\left(\alpha_{1}+\beta_{2}+\beta_{3}\right) & 0 & \alpha_{3} & 0 & \alpha_{2} & 0 \\
\beta_{2} & 0 & -\left(\alpha_{2}+\beta_{2}+\beta_{3}\right) & 0 & 0 & \alpha_{1} & \alpha_{3} \\
0 & \beta_{3} & 0 & -\left(\alpha_{1}+\alpha_{3}\right) & \beta_{1} & 0 & 0 \\
\beta_{3} & 0 & 0 & \alpha_{1} & -\left(\alpha_{3}+\beta_{1}\right) & 0 & 0 \\
0 & \beta_{2} & \beta_{1} & 0 & 0 & -\left(\alpha_{1}+\alpha_{2}\right) & 0 \\
0 & 0 & \beta_{3} & 0 & 0 & 0 & -\alpha_{3}
\end{array}\right]\left[\begin{array}{l}
P_{0} \\
P_{1} \\
P_{2} \\
P_{3} \\
P_{4} \\
P_{5} \\
P_{6}
\end{array}\right]=\left[\begin{array}{l}
0 \\
0 \\
0 \\
0 \\
0 \\
0 \\
0
\end{array}\right]
$$

Using the following normalizing condition

$$
P_{0}(\infty)+P_{1}(\infty)+P_{2}(\infty)+P_{3}(\infty)+P_{4}(\infty)+P_{5}(\infty)+P_{6}(\infty)=1
$$

We substitute (5) in any of the redundant rows in (4) to give

$$
\left[\begin{array}{ccccccc}
-\left(\beta_{1}+\beta_{2}+\beta_{3}\right) & \alpha_{1} & \alpha_{2} & 0 & \alpha_{3} & 0 & 0 \\
\beta_{1} & -\left(\alpha_{1}+\beta_{2}+\beta_{3}\right) & 0 & \alpha_{3} & 0 & \alpha_{2} & 0 \\
\beta_{2} & 0 & -\left(\alpha_{2}+\beta_{2}+\beta_{3}\right) & 0 & 0 & \alpha_{1} & \alpha_{3} \\
0 & \beta_{3} & 0 & -\left(\alpha_{1}+\alpha_{3}\right) & \beta_{1} & 0 & 0 \\
\beta_{3} & 0 & 0 & \alpha_{1} & -\left(\alpha_{3}+\beta_{1}\right) & 0 & 0 \\
0 & \beta_{2} & \beta_{1} & 0 & 0 & -\left(\alpha_{1}+\alpha_{2}\right) & 0 \\
1 & 1 & 1 & 1 & 1 & 1 & 1
\end{array}\right]\left[\begin{array}{c}
P_{0}(\infty) \\
P_{1}(\infty) \\
P_{2}(\infty) \\
P_{3}(\infty) \\
P_{4}(\infty) \\
P_{5}(\infty) \\
P_{6}(\infty)
\end{array}\right]=\left[\begin{array}{l}
0 \\
0 \\
0 \\
0 \\
0 \\
0 \\
1
\end{array}\right]
$$

We solve for the system of equations in the matrix above to obtain the steady-state probabilities

$$
P_{0}(\infty), P_{1}(\infty), P_{2}(\infty), P_{7}(\infty), P_{4}(\infty)
$$

The steady-state availability is given by

$$
A(\infty)=\frac{\alpha_{1} \alpha_{2} \alpha_{3}+\alpha_{2} \alpha_{3} \beta_{1}+\alpha_{1} \alpha_{2} \beta_{2}+\alpha_{1} \alpha_{2} \beta_{3}}{\alpha_{1} \alpha_{3} \beta_{2}+\alpha_{1} \alpha_{2} \beta_{3}+\alpha_{1} \alpha_{2} \alpha_{3}+\alpha_{1} \beta_{2} \beta_{3}+\alpha_{2} \beta_{1} \beta_{3}+\alpha_{2} \alpha_{3} \beta_{1}+\alpha_{3} \beta_{1} \beta_{2}}
$$

\subsection{Busy Period Analysis}

Using the same in itial condition as for the reliability case:

$$
P(0)=\left[P_{0}(0), P_{1}(0), P_{2}(0), P_{3}(0), P_{4}(0), P_{5}(0), P_{6}(0)\right]=[1,0,0,0,0,0,0]
$$

The differential equations can be expressed as

$$
\left[\begin{array}{c}
\dot{P_{0}} \\
\dot{P_{1}} \\
\cdot \pm \\
P_{2} \\
\dot{P_{3}} \\
\dot{P_{4}} \\
\dot{P_{5}} \\
\dot{P_{6}}
\end{array}\right]=\left[\begin{array}{ccccccc}
-\left(\beta_{1}+\beta_{2}+\beta_{3}\right) & \alpha_{1} & \alpha_{2} & 0 & \alpha_{3} & 0 & 0 \\
\beta_{1} & -\left(\alpha_{1}+\beta_{2}+\beta_{3}\right) & 0 & \alpha_{3} & 0 & \alpha_{2} & 0 \\
\beta_{2} & 0 & -\left(\alpha_{2}+\beta_{2}+\beta_{3}\right) & 0 & 0 & \alpha_{1} & \alpha_{3} \\
0 & \beta_{3} & 0 & -\left(\alpha_{1}+\alpha_{3}\right) & \beta_{1} & 0 & 0 \\
\beta_{3} & 0 & 0 & \alpha_{1} & -\left(\alpha_{3}+\beta_{1}\right) & 0 & 0 \\
0 & \beta_{2} & \beta_{1} & 0 & 0 & -\left(\alpha_{1}+\alpha_{2}\right) & 0 \\
0 & 0 & \beta_{3} & 0 & 0 & 0 & -\alpha_{3}
\end{array}\right]\left[\begin{array}{c}
P_{0} \\
P_{1} \\
P_{2} \\
P_{3} \\
P_{4} \\
P_{5} \\
P_{6}
\end{array}\right]
$$

In the steady state, the derivatives of the state probabilities become zero and this will enable us to compute steady state busy : 


$$
B(\infty)=1-P_{0}(\infty)
$$

and

$A P=0$, which in matrix form

$$
\left[\begin{array}{ccccccc}
-\left(\beta_{1}+\beta_{2}+\beta_{3}\right) & \alpha_{1} & \alpha_{2} & 0 & \alpha_{3} & 0 & 0 \\
\beta_{1} & -\left(\alpha_{1}+\beta_{2}+\beta_{3}\right) & 0 & \alpha_{3} & 0 & \alpha_{2} & 0 \\
\beta_{2} & 0 & -\left(\alpha_{2}+\beta_{2}+\beta_{3}\right) & 0 & 0 & \alpha_{1} & \alpha_{3} \\
0 & \beta_{3} & 0 & -\left(\alpha_{1}+\alpha_{3}\right) & \beta_{1} & 0 & 0 \\
\beta_{3} & 0 & 0 & \alpha_{1} & -\left(\alpha_{3}+\beta_{1}\right) & 0 & 0 \\
0 & \beta_{2} & \beta_{1} & 0 & 0 & -\left(\alpha_{1}+\alpha_{2}\right) & 0 \\
0 & 0 & \beta_{3} & 0 & 0 & 0 & -\alpha_{3}
\end{array}\right]\left[\begin{array}{c}
P_{0} \\
P_{1} \\
P_{2} \\
P_{3} \\
P_{4} \\
P_{5} \\
P_{6}
\end{array}\right]=\left[\begin{array}{l}
0 \\
0 \\
0 \\
0 \\
0 \\
0 \\
0
\end{array}\right]
$$

We solve for $P_{0}(\infty)$

Using the following normalizing condition

$$
P_{0}(\infty)+P_{1}(\infty)+P_{2}(\infty)+P_{3}(\infty)+P_{4}(\infty)+P_{5}(\infty)+P_{6}(\infty)=1
$$

We substitute (7) in any of the redundant rows in (4) to give

$$
\left[\begin{array}{ccccccc}
-\left(\beta_{1}+\beta_{2}+\beta_{3}\right) & \alpha_{1} & \alpha_{2} & 0 & \alpha_{3} & 0 & 0 \\
\beta_{1} & -\left(\alpha_{1}+\beta_{2}+\beta_{3}\right) & 0 & \alpha_{3} & 0 & \alpha_{2} & 0 \\
\beta_{2} & 0 & -\left(\alpha_{2}+\beta_{2}+\beta_{3}\right) & 0 & 0 & \alpha_{1} & \alpha_{3} \\
0 & \beta_{3} & 0 & -\left(\alpha_{1}+\alpha_{3}\right) & \beta_{1} & 0 & 0 \\
\beta_{3} & 0 & 0 & \alpha_{1} & -\left(\alpha_{3}+\beta_{1}\right) & 0 & 0 \\
0 & \beta_{2} & \beta_{1} & 0 & 0 & -\left(\alpha_{1}+\alpha_{2}\right) & 0 \\
1 & 1 & 1 & 1 & 1 & 1 & 1
\end{array}\right]\left[\begin{array}{l}
P_{0}(\infty) \\
P_{1}(\infty) \\
P_{2}(\infty) \\
P_{3}(\infty) \\
P_{4}(\infty) \\
P_{5}(\infty) \\
P_{6}(\infty)
\end{array}\right]=\left[\begin{array}{l}
0 \\
0 \\
0 \\
0 \\
0 \\
0 \\
1
\end{array}\right]
$$

The steady state busy period $B(\infty)$ is therefore

$$
B(\infty)=\frac{\alpha_{1} \alpha_{2} \beta_{2}+\alpha_{1} \alpha_{2} \beta_{3}+\alpha_{1} \beta_{2} \beta_{3}+\alpha_{2} \beta_{1} \beta_{3}+\alpha_{2} \alpha_{3} \beta_{1}+\alpha_{3} \beta_{1} \beta_{2}}{\alpha_{1} \alpha_{3} \beta_{2}+\alpha_{1} \alpha_{2} \beta_{3}+\alpha_{1} \alpha_{2} \alpha_{3}+\alpha_{1} \beta_{2} \beta_{3}+\alpha_{2} \beta_{1} \beta_{3}+\alpha_{2} \alpha_{3} \beta_{1}+\alpha_{3} \beta_{1} \beta_{2}}
$$

\subsection{Profit analysis}

Following[1,3]the expected profit per unit time incurred to the system in the steady-state is given by:

Profit =total revenue fro $\mathrm{m}$ system using - total cost due to repair

$$
\mathrm{PF}=R A(\infty)-C_{1} B(\infty)
$$

Where PF: is the profit incurred to the system $\mathrm{R}$ : is the revenue per unit up time of the system

$C_{1}$ : is the cost per unit time which the system is under repair

\section{Results}

Fig. 2: shows relation between type I repair rate and MTSF of the system

Fig. 3: shows relation between type I repair rate and availability of the system

Fig. 4: shows the relation between type I repair rate and profit function of the system

Table 1: shows the relation between type I repair rate and the profit of the system.

\section{Discussion and Conclusions}

\subsection{Discussion}

For the study of system behavior, we plot graphs in Fig. 2 to 4 for MTSF, system availability and profit function with respect to $\alpha_{1}$ while other parameters are kept fixed as $\alpha_{2}=0.5, \alpha_{3}=0.002, \beta_{1}=0.5, \beta_{2}=0.06, \beta_{3}=0.01$, $R=1000, C_{1}=100$,

From Fig. 2 it is clear that MTSF increases with increase in the value of $\alpha_{1}$ which reflects the effect of perfect repair on MTSF. From Fig. 3 the system availability increases with increase in the value of $\alpha_{1}$. Thus, the more the repair is perfect, the more the system is available for operation. In Fig. 4 it can be observed that with increase in the value of $\alpha_{1}$ the profit also increase. Thus, the more the system is in perfect condition the more it is effective. Same argument can also be observe in table 1 where increase in the value of $\alpha_{1}$ lead to the increase in profit function. 
Table 1. Relation between type I repair rate and the profit

\begin{tabular}{|c|c|}
\hline$\alpha_{1}$ & Profit \\
\hline 0 & 63.40 \\
\hline 0.1 & 200.64 \\
\hline 0.2 & 296.00 \\
\hline 0.3 & 366.12 \\
\hline 0.4 & 419.83 \\
\hline 0.5 & 462.31 \\
\hline 0.6 & 496.73 \\
\hline 0.7 & 525.19 \\
\hline 0.8 & 549.12 \\
\hline 0.9 & 569.52 \\
\hline 1 & 587.12 \\
\hline
\end{tabular}

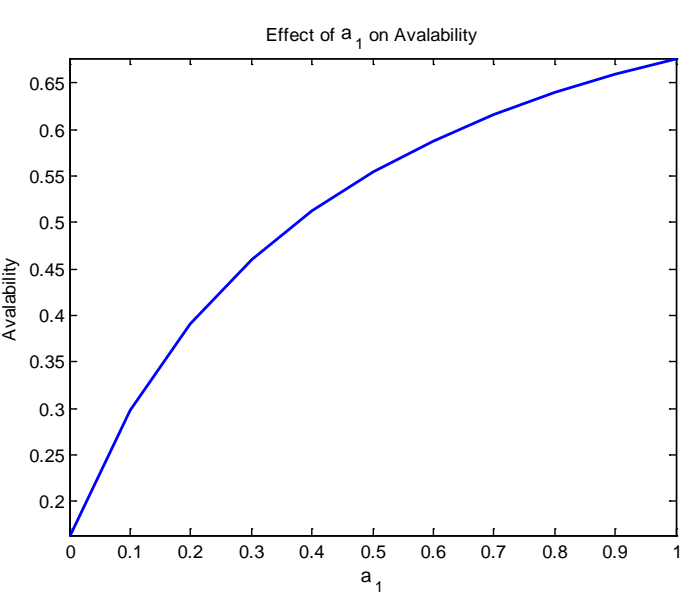

Figure 3. effect of type I repair rate on system availability

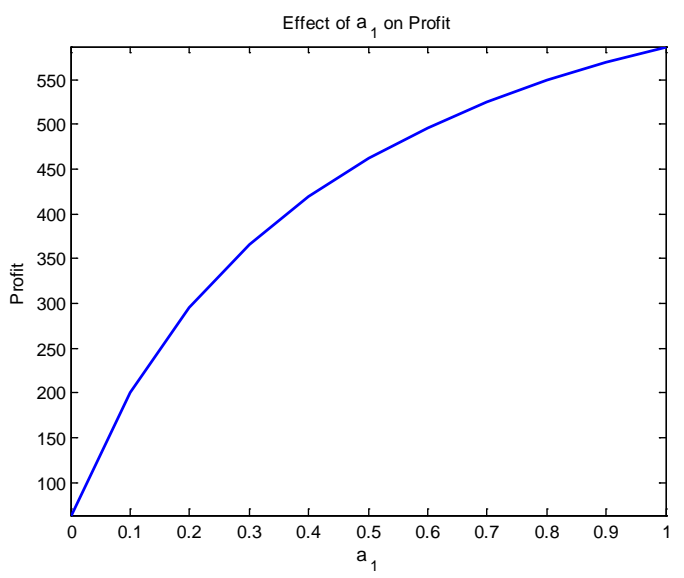

Figure 4. effect of type I repair rate on profit function

\subsection{Conclusions}

In this study, we developed the explicit expressions for MTSF, system availability, busy period and profit function for 2-out-of-3 system subjected to perfect repair. Graphs were plotted to show the effect of perfect repair on important measures of system effectiveness. Simulations results show that perfect repair is important particularly in increasing mean time to system failure, availability, and profit function and system performance as a whole.

\section{REFERENCES}

[1] El-Said, K.M.,. ( 2008).Cost analysis of a system with preventive maintenance by using Kolmogorov's forward equations method. Ame. J. of App. Sci. 5(4), 405-410

[2] Haggag, M.Y., (2009). Cost analysis of a system involving common cause failures and preventive maintenance.J. Maths. And Stat. 5(4), 305-310

[3] Haggag, M.Y., (2009). Cost analy sis of k-out-of-n repairable system with dependent failure and stand by support using kolmogorov's forward equations method. J. Maths. And Stat. 5(4), 401-407

[4] El-Said, K.M., and M.S. El-Sherbeny. ( 2005).Profit analy sis of a two unit cold standby system with preventive maintenance and random change in units. J. Maths and Stat.,1 (1) $71-77$

[5] El-Said, K.M., and M.S. El-Sherbeny. (2005) .Evaluation of reliability and availability characteristics of two different systems by using linear first order differential equations. J. Maths and Stat.,1 (2) 119-123

[6] Gupta., R. and Mittal., M. (2006). Stochastic analysis of a compound redundant system involving human failure. J. Maths and Stat. 2 (3), pp 407-413.

[7] Wang, K., Hseih, C., and Liou, C. H. (2006). Cost benefit analysis of series systems with cold standby components and a repairable service station. Quality technology and quantitative management. Vol. 3(1), pp 77-92.

[8] Bhatti, J., Chitkara, A., and Bhardwaj, N., (2011). Profit analysis of two unit cold standby system with two types of failures under inspection policy and discreet distribution. International Journal of science and engineering research, Vol.2 (2), pp 1-7.

[9] Kumar, Jitender., Kady an, M.S. and Malik, S.C., (2010). Cost benefit analysis of a two unit parallel system subject to degradation after repair. Applied mathematical sciences, 4(56), pp 2749-2758.

[10] Gupta, R., Goel, C.K. and Tomer, A. (2010). Analysis of a two unit standby system with correlated failure and repair nd random appearance and disappearance of repairman.

[11] Malik, S.C., Bharwaj, R.K. and Grewal, A.S. (2010). Probabilistic analysis of a system of two non identical parallel units with priority to repair to repair subject to inspection. Journal of reliability and statistical studies, vol. 3(1), pp 1-11

[12] Mahmoud, M.A.W. and Moshref, M.E. (2010). On a two unit cold standby system considering hardware, human error failures and preventive maintenance, Mathematics and Computer modeling, 51(5-6), pp 736-745.

[13] Yusuf, I. and Bala, S.I. (2012). Stochastic modeling of a two unit parallel system under two types of failures. International Journal of Latest trends in Mathematics, Vol. 2 (1), pp 44-53

[14] Kumar, J. and Kadyan, M.S. (2012). Profit analysis of a system of non identical units with degradation and replacement. International journal of computer application, vol. 40 (3), pp 19-25

[15] Sureria, J.K., Malik, S.C. and Anand, J. (2012). Cost benefit analysis of a computer system with priority to software replacement over hardware repair. Applied Mathematical Sciences, vol. 6 (75), pp 3723-3734. 